\title{
Diversidad sorda y violencia de género: restricción comunicativa en el uso de la lengua de señas en salud
}

\author{
Gina V. Morales-Acosta, Aura I. Aguilar-Caro
}

Resumen. En este trabajo se reflexiona sobre la diversidad sorda, la violencia de género y la comunicación, al discutir la necesidad de políticas lingüísticas inclusivas que permitan la adquisición y el desarrollo de la lengua de señas y su uso desde la primera infancia, en relación comunicativa intercultural que se origina entre una cultura oyente y una cultura sorda. Las mujeres sordas presentan mayor grado de 'violencia obstétrica', que involucra a los profesionales implicados en la atención directa de la salud sexual y reproductiva, en algunos casos por no contar el personal de salud con elementos comunicativos en lengua de señas básicos como resultado de una hegemonía dominante de la lengua oral. En este sentido se identifica el uso de un solo formato comunicativo, la lengua (oral), con procedimientos médicos no informados o en acciones sin puentes comunicativos, que revelan una restricción comunicativa para una plena información y conocimiento, necesarios en diferentes niveles de contextos sociales.

Palabras clave. Diversidad sorda. Género. Lengua de señas. Violencia.

\section{Deaf diversity and gender violence: restricted communication in the use of sign language in health}

Summary. This paper aims to reflect on deaf diversity and gender violence, to discuss language policies for the acquisition and development of sign language and its use, in social contexts in a dominant hegemony of oral language. The communicative access of women and girls that is possible between a hearing culture and a deaf culture, in full access, without restriction to information and knowledge. Deaf women have a higher degree of 'obstetric violence' that involves professionals involved in the direct care of sexual and reproductive health, in some cases because health personnel do not have communicative elements in basic sign language, in uninformed medical procedures or in actions taken without communicative bridges.

Key words. Deaf diversity. Gender. Sign language. Violence.

\section{Introducción}

Al abordar la diversidad, inherente al ser humano, en el foco del presente artículo se encontrará la comunicación, y nos referiremos a las personas sordas que han nacido en una comunidad sorda y a la adquisición de la lengua de señas que se da de forma natural en la primera infancia, como su lengua materna, o aprendida posteriormente en la etapa escolar en la escuela.

Por otro lado, en contextos sociales de una hegemonía dominante de la lengua oral, se encuentran los que provienen de un entorno oyente y han sido oralizados mediante métodos bimodales, como el cued speech o la palabra complementada.

En este orden de ideas, se define la diversidad sorda [1] como las diferentes formas de comunicarse (lengua de señas o lengua oral) que acogen a los dos grupos en: a) lengua materna, como la lengua de señas, su adquisición y desarrollo que se originan con interlocutores nativos de la lengua o bilingües y no en terapias; b) lengua oral, o bimodal, como el uso simultáneo de algunos apuntadores de lengua de señas y expresiones orales, cued speech o palabra complementada, aprendidos en rehabilitación o terapia, usados por tradición en la educación de los sordos como métodos comunicativos funcionales.

Por lo anterior, las problemáticas comunicativas de ambos grupos son totalmente distintas. No se pueden tratar de forma homogénea para seguir las orientaciones $\mathrm{u}$ explicaciones que un interlocutor oyente les pueda dar a través de la lengua oral.

La violencia comunicativa, en referencia a la restricción lingüística o comunicativa, la experimentan hombres y mujeres de diversidad sorda, ejercida en lo más básico de la condición humana, el lenguaje: por un lado, como asimilación, donde las personas sordas se ven obligadas a comunicarse en len-
Facultad de Ciencias de la Salud; Núcleo de Investigación de Docencia del Departamento de Ciencias de la Rehabilitación y Movimiento Humano; Universidad de Antofagasta; Antofagasta, Chile (G.V. MoralesAcosta). Universidad Simón Bolívar; Barranquilla, Colombia (A.I. AguilarCaro).

Correspondencia:

Dra. Gina Viviana Morales Acosta. Facultad de Ciencias de la Salud. Universidad de Antofagasta. Angamos, 601. CP 02800. Antofagasta, Chile.

E-mail: gina.morales@uantof.cl.

Nota:

La escritura de 'Sorda/o' (con S mayúscula) devela la enunciación y posicionamiento comunicativo en la reivindicación de la lengua de señas y respeto a la cultura sorda (Woodward, 1972), como punto de enunciación del presente escrito. No obstante, ateniéndose a las normativas generales de la Real Academia Española, en esta publicación aparece 'sorda/o' en minúscula.

Recibido:

31.05.18.

Aceptado:

16.07.18.

Conflicto de intereses: No declarado.

Competing interests: None declared.

(C) 2018 FEM 
gua oral, dado que los profesionales desconocen la lengua de señas; y por otro, como agresión física y cultural en propuestas clínicas orientadas al cuerpo y su funcionamiento para 'normalizar', a través de una ayuda técnica de cirugía de implante coclear o prótesis auditivas (audífono), sin una alternativa de comunicación clara con relación a la lengua materna, como lo es la lengua de señas.

Abordar la diversidad sorda, usuaria de la lengua de señas, problematiza la denominación de personas en situación de discapacidad, donde encontramos en el lenguaje apuntadores de discriminación de 'menos válido'. Estos elementos aumentan en el ámbito de la violencia de género [2]. Se extrapola esta situación social en la identidad femenina, que coloca en desventaja a mujeres y niñas, en situación de discapacidad (no sólo auditiva).

Esto lleva a reflexionar desde los derechos humanos y desde el acceso a la comunicación, como sujetos de derechos, para buscar alternativas simétricas desde la educación que permitan hacer planteamientos de inclusión.

Las estadísticas de discapacidad y sus concepciones para recoger datos [3,4] varían según las políticas públicas de los países. Encontramos en el informe presentado por la Comisión Económica para América Latina y el Caribe que: '[...] es posible estimar que, en la actualidad, alrededor del $12 \%$ de la población de América Latina y el Caribe viviría al menos con una discapacidad, lo que involucraría aproximadamente a 66 millones de personas' [5].

En la anterior información hallamos brechas de participación de la fuerza de trabajo en la región [6], y vemos que, en la diversidad sorda, las mujeres en edad productiva están desempleadas o con empleos informales, lo que no les permite mejorar la calidad de vida.

\section{Lenguaje y violencia}

Los derechos lingǘsticos [7], entendidos como el 'derecho que cada comunidad lingüística tiene para dar forma a la vida en su lengua propia y en todos los ámbitos', otorgan legitimidad a lo más básico del ser humano y la dignidad humana al poder comunicarse, en ámbitos del lenguaje comprensivo y expresivo, en su lengua materna.

La lengua de señas ha vivenciado diferentes formas de enunciación en pro de su reconocimiento, como 'lenguaje mímico, 'lenguaje manual, 'lenguaje gestual', 'lenguaje signado' o 'lenguaje de signos manuales'; por ejemplo, en España se utiliza la de lenguaje de signos o lengua de señas.
Lingüistas relacionados con el área referencian en los textos la traducción anglosajona de sing linguistics y sing language por 'lengua de señas', y este último es el término adoptado y usado en algunos países de Latinoamérica, en particular en Chile, donde se reconoce el lenguaje de señas chileno (en la ley) haciendo alusión a la lengua.

Se acota que la lengua de señas no es universal y que posee estructuras sintácticas en constante desarrollo inherentes a una lengua y gramática propias [8] que permiten la representación del mundo con las manos, con características visuogestuales de la lengua.

La lengua de señas no se adquiere de forma espontánea, requiere locutores o hablantes nativos y bilingües [1]: 'la adquisición y desarrollo de la lengua de señas no se da de manera espontánea, requiere un proceso de aprendizaje [en la primera infancia], para una posterior mediación comunicativa en el colegio con modelos lingüísticos sordos o profesores bilingües.'

Con relación al ingreso de las niñas en la escuela, en ocasiones la escolaridad se da en momentos tardíos, o con deserción, pues se enfrentan a ser cuidadoras, heteroasignadas, en trabajos y oficios básicos [1] para generar el sustento del hogar.

Por su parte, el acceso a la información en la lengua materna, en este caso la lengua de señas, es restringido, es decir, se realiza violencia comunicativa al no poder ejercer el derecho lingüístico [7]. Esta violencia se justifica al enfrentarse a un bajo acceso de la múltiple información existente en el área a través de la lengua de señas, que podría ser mejorada en vinculación con medios comunicativos accesibles como 'centro de relevo', intérpretes profesionales formados, o en algo tan básico como los programas de televisión con close caption.

Para la atención en el ámbito de la salud [9], son las mujeres sordas las que presentan un mayor grado de 'violencia obstétrica', que involucra a los profesionales implicados en la atención directa de la salud sexual y reproductiva, por no contar el personal de salud con elementos comunicativos básicos en lengua de señas, en procedimientos médicos no informados o en acciones tomadas por no tener puentes comunicativos, como, por ejemplo, la atención del parto por cesárea [10], o diagnósticos que la familia o menores de edad deben comunicar a las mujeres sordas que consultan.

En relaciones comunicativas en eventos cotidianos, como, por ejemplo, en viajes aéreos, la diversidad sorda se enfrenta a hechos reiterativos en el aeropuerto (nacional e internacional), como anunciar los cambios del vuelo o la sala de espera en el 
altavoz, sin considerar o tener un centro de información en lengua de señas ni un vídeo informativo.

\section{Identidad sorda}

Las diferentes polifonías que convergen en el abordaje de la diversidad, como un conjunto de atributos identitarios que se relacionan de una forma marcada con la estética del cuerpo y con su funcionalidad, parten de la idea de que 'las representaciones que se crean en relación con las personas con discapacidad insisten en remarcar justamente la discapacidad (la dolencia, el síndrome, la deformidad, la carencia, lo que le falta) y no la persona en sí' [11].

Hay representaciones sociales que se constituyen en actos de violencia y discriminación, ante las cuales se busca resarcirse a través de la agenda 2030 [12]. El objetivo número 5, 'Lograr la igualdad entre los géneros y empoderar a todas las mujeres y las niñas', se explicita en 'Poner fin a todas las formas de discriminación contra todas las mujeres y las niñas en todo el mundo' (indicador 5.1).

Como se menciona en la agenda 2030, de este objetivo se derivan acciones orientadas a poner fin a la discriminación, la violencia en lo público o privado, la trata de explotación sexual y otros tipos de explotación, el matrimonio infantil forzado, la mutilación genital femenina y el trabajo doméstico no remunerado, y a promover el liderazgo, el acceso a la salud sexual y reproductiva y sus derechos, la igualdad de derechos de recursos económicos, el uso de la tecnología y las leyes de empoderamiento de las mujeres y niñas en todos los niveles.

Como consecuencia de lo anterior, en relación con situaciones que incluyan la violencia de género en migración y diversidad, en declaraciones realizadas por la Organización Internacional para las Migraciones [13], no se podrá garantizar la migración segura, ordenada y humana sin que se considere la perspectiva de género.

Esto, por su parte, lo ha ratificado la Organización Internacional del Trabajo [14], que considera que un trabajo de tiempo completo coarta a las cuidadoras de personas en situación de discapacidad [15], es decir, las familias de niñas sordas deben entrenarse en el aprendizaje de la lengua de señas, como se mencionó anteriormente.

Las mujeres en el trabajo [14] menciona directrices para contratar a personas en situación de discapacidad y dice que la remuneración debe estar dentro de la escala salarial, es decir, que deben recibir un pago de igual valor.

\section{Identidad de género y exclusión}

Concatenado con lo anterior, la doble o triple exclusión que integran las mujeres desde su identidad, sumadas a otras situaciones particulares en el ámbito físico, étnico, social, religioso y político, existe desde que se ha conformado la humanidad misma. En una hegemonía heteronormativa patriarcal se reivindica, en este caso, en torno a la diversidad sorda, la necesidad de legitimar la lengua materna (la lengua de señas).

Lo anterior es un factor clave para comprender la historicidad en la exclusión que viven en la cotidianidad mujeres y niñas (y varones), con toda la deuda histórica que ha existido en respeto a la lengua. En las conclusiones del Congreso de Milán [16] se hizo necesario reconocer, en un acto de resistencia, las demandas de las asociaciones de sordos que indican que la lengua de señas deben enseñarla locutores nativos sordos, como modelos lingüísticos portadores del acervo cultural de la comunidad sorda.

La reivindicación de la inclusión de la mitad del género humano en los principios universales de la ilustración, y, a su vez, la aplicación del principio de igualdad, la educación y la emancipación de los prejuicios, constituyen los principales pilares de la obra clásica de Wollstonecraft [17], publicada en 1792 en Inglaterra, a partir de los cuales se puede ubicar una parte importante de los inicios de movimientos de mujeres.

En la modernidad, la obsesión patriarcal de la legitimación se encuentra en una serie de supuestos que ya no hacen posible su configuración como obsesión genealógica. Precisamente, se lucha contra la tradición, contra lo heredado, contra la idea aristocrática de linaje, contra el régimen. La relevancia de la mujer en la genealogía aparece solamente como un agente distorsionante o perturbador, causante de uniones no controladas por los pactos entre varones: uniones contra natura, a mala distancia [18].

Pateman y Agra [19] dan a entender que la humanidad tiene dos cuerpos diferenciados y que las mujeres deben acceder a la ciudadanía y a la democracia como mujeres, es decir, deben incorporarse como individuos concretos, corpóreos, dotados de una capacidad diferente -la capacidad de dar a luz, de crear vida-, y no como individuos abstractos, descorporeizados, masculinos, pues se ocultan las relaciones de poder y la subordinación de las mujeres, con el objetivo ya no de reconstruir la tradición, sino de pensar en alguna alternativa para situarse en una ciudadanía genuinamente democrática.

En ese sentido, la mujer no se reivindica como sujeto, porque carece de los medios concretos para 
ello, porque experimenta el lazo necesario que la une al hombre sin plantearse reciprocidad alguna, y porque a menudo se complace en su papel de otro. A un hombre no se le ocurriría escribir un libro sobre la singular situación que ocupan los varones en la humanidad. Si quiero definirme, quiero antes que nada declarar 'soy una mujer.' Un hombre no piensa jamás en presentarse como individuo de un determinado sexo: que él sea hombre se da por supuesto. Es sólo de una manera formal, en los registros de las alcaldías y las declaraciones de identidad, donde las rúbricas de masculino y femenino aparecen como simétricas [20].

Desde los años setenta hasta los noventa, el movimiento feminista agitó la consciencia colectiva y espoleó a la opinión pública dando nombre a realidades que, a pesar de que tenían mucha historia, permanecían invisibles para la mayoría de la población. Los conceptos de género y patriarcado se abren paso en este período de tiempo con algunos mensajes políticos e intelectuales inequívocos. En efecto, se conceptualiza la categoría género como una construcción social e histórica que revela la profunda desigualdad entre varones y mujeres, pues señala dos normatividades, la masculina y la femenina, construidas asimétricamente [21].

Cuando ya en la década de los setenta comenzó a adoptarse la denominación de 'género', se propuso una analogía que se tornó clásica: la naturaleza es a la cultura como el sexo es al género, inscribiendo el género claramente en el ámbito de la cultura y haciéndolo depender de ella. Más adelante, a finales de la década de los ochenta, desde la academia estadounidense se fue imponiendo la dupla identitaria sexo-género entendida como inestable, en constante modificación, aunque siempre ligada a contextos culturales como parte importante del proceso mismo de producción de sexo-género. La feminista Judith Butler entiende que tanto el sexo como el género son lábiles y resignificables en el constante ejercicio paradójico de la sexualidad [22].

Otro logro significativo, además de la comprensión de la noción de 'género y desarrollo', desde lo teórico, a partir del año 1995, en la cuarta conferencia de la mujer en Pekín, es que se introduce la categoría 'género' para describir las características de los hombres y las mujeres que son determinadas y construidas socialmente; de ese concepto 'género' surge lo que hoy se conoce como 'perspectiva de género', que es una herramienta para comprender cómo estas construcciones adjudicadas a lo femenino y a lo masculino afectan o impactan en determinados procesos.

$\mathrm{Al}$ analizar las diferencias existentes en las construcciones sociales adjudicadas a lo femenino y a lo masculino y la relación que establecen entre ellos, podemos ubicar problemas de una manera más eficaz [17]. De ahí que se potencie a partir de la conferencia de Pekín la transversalización del género y se inste a los gobiernos a hacerla parte de la política pública mundial.

Es así como todos los estudios que integren la categoría femenina en la actualidad son fundamentales, ya que intrínsecamente hay historias acumuladas con lo femenino que es necesario resarcir desde lo investigativo; ya no sólo con las categorías excluyentes meramente por ser mujer, sino con lo subyacente como sujeto histórico, con sus limitaciones, aunque como investigadoras buscar desde el conocimiento la manera de vincularlas a las nuevas formas de estar en lo contemporáneo.

\section{Comunicación y violencia}

Por una parte, en la sordedad se reafirma la importancia de la lengua como herramienta de acceso a la cultura y la construcción de la identidad [23], con relevancia comunicativa en todos los contextos sociales.

Por otra parte, el informe sobre el 'Estado Mundial de la Infancia 2013: niñas y niños con discapacidad' [24] pone en evidencia la mayor desventaja de las niñas. En el informe se afirma que 'el género es un factor clave, ya que las niñas con discapacidad tienen menos probabilidades que los niños de recibir alimentos y atención', y agrega que 'la discriminación por motivos de discapacidad es una forma de opresión', para concluir que '[...] múltiples carencias conducen a una mayor exclusión para muchos niños/as con discapacidad'.

Es así como en el lenguaje, junto con la lengua herramientas de construcción de la identidad, se encuentran los apuntadores de discriminación y, al no compartir una lengua que permita negociación e información, se propician actos de violencia, como el no reconocimiento de los derechos humanos. Esta situación se da desde el mismo momento en que en la niñez no se reconoce la diversidad en torno a la discapacidad o pertenencia étnica y, por tanto, no se logra armonizar en el lenguaje y la correspondiente lengua de una comunidad.

\section{Conclusiones}

En la diversidad sorda, para la adquisición y el desarrollo de la lengua de señas se requieren políticas lingüísticas inclusivas, con una financiación adecuada y sostenible, que permitan abarcar diferentes 
contextos sociales y propendan a erradicar la violencia comunicativa en el acceso a la información y el conocimiento.

La violencia comunicativa revela la infantilización de las mujeres en diferentes situaciones, que pueden darse cuando asisten a una consulta médica o como asistentes (apoderadas) a reuniones del colegio sin intérprete en lengua de señas.

Se precisa replantear la heterodesignación para fortalecer el poder acceder a la educación con calidad en edad temprana, con docentes bilingües, donde no sólo orientar la enseñanza para las mujeres y niñas a oficios manuales, alejados de la educación regular.

Reconocemos que el manejo de la lengua de señas en la sociedad conduce a una autonomía en la interacción comunicativa y en la información en la lengua de señas en otros espacios, como eventos académicos, salud, recreación, ocio y tiempo libre, y acciones financieras, entre otros.

Las acciones de la agenda 2030 en salud inclusiva, lingüística y de género tienden a una atención en salud que permita un desarrollo y a lograr 'poner fin a todas las formas de discriminación contra todas las mujeres y las niñas en todo el mundo.'

Es de vital importancia analizar la situación actual en atención en salud de los hombres y las mujeres con diversidad sorda usuarios de la lengua de señas, que permita para estas últimas disminuir la violencia obstétrica al recibir procedimientos informados y consensuados; y desarrollar estrategias de seguimiento para situaciones comunicativas interculturales, las cuales se dan entre una cultura oyente y una cultura sorda, que faciliten el pleno acceso a la información en la promoción de la salud y la prevención de la enfermedad.

Se debe propiciar de forma constante, con el personal de salud, el aprendizaje y el conocimiento de la lengua de señas, en respeto comunicativo que oriente la inclusión en la sordedad.

\section{Bibliografía}

1. Morales G. Diversidad auditiva: imaginarios sociales e inclusión laboral: una aproximación. Psicogente 2015; 18: 364-71.
2. Soler A, Teixeira T, Jaime V. Discapacidad y dependencia: una perspectiva de género. XI Jornadas de Economía Crítica. Bilbao, 27-29 de marzo de 2008.

3. Cruz R. Representaciones sobre discapacidad y educación: referentes a partir de los datos estadísticos. Pasaje 2016; 2 : 67-91.

4. Barton L. Estudios sobre discapacidad y la búsqueda de inclusividad: observaciones. Red de Educación 2015; 3: 137-51.

5. Comisión Económica para América Latina y el Caribe (CEPAL). Panorama social de América Latina y el Caribe (1). Santiago de Chile: ONU; 2012.

6. Organización Internacional del Trabajo (OIT). Perspectivas Sociales y del Empleo en el Mundo (2016). URL: http://www. ilo.org/wcmsp5/groups/public/---dgreports/---dcomm/--publ/documents/publication/wcms_443505.pdf. [01.04.2018].

7. Unesco. Declaración universal de los derechos lingüísticos. Barcelona: Institut d'Edicions de la Diputació de Barcelona; 1998.

8. Oviedo A. Apuntes para una gramática de la lengua de señas colombiana. Bogotá: Mineduc-Insor; 2001.

9. Dagron J. Représentations sociales de la surdité. Psychologie de la Surdité 2006; 3: 239-52.

10. Dagron J. Sourds et soignants, deux mondes, une médecine. Paris: Éditions In Press; 1999.

11. Campos M. Identidades 'anormais': (des) construção dos corpos 'deficientes' 29a. Reunião Anual da ANPED; 2006.

12. Unesco. Objetivos de desarrollo sostenible 2015-2030 (ODS). Unesco; 2015.

13. Organización Internacional para las Migraciones (OIM). Políticas de la OIM sobre igualdad de género (2015-2019). URL: https://www.iom.int/sites/default/files/about-iom/gender/ C-106-INF-8-Rev.1-Pol\%C3\%ADtica-de-la-OIM-sobre-laIgualidad-de-G\%C3\%A9nero-2015-2019.pdf. [01.04.2018].

14. Organización Internacional del Trabajo (OIT). Las mujeres en el trabajo, tendencias (2016). URL: http://www.oitcinterfor. org/node/6794. [01.04.2018].

15. Hein C, Cassirer N. Soluciones para el cuidado infantil en el lugar de trabajo. Madrid: Ministerio de Trabajo e Inmigración; 2010.

16. Oviedo A. El congreso de Milán, 1880 (2006). URL: http:// www.cultura-sorda.org/el-2do-congreso-internacional-demaestros-de-sordomudos-milan-1880/. [01.04.2018].

17. Wollstonecraft $M$. Vindicación de los derechos de la mujer. Madrid: Akal; 2005.

18. Amorós C. Crítica de la razón patriarcal. Barcelona: Anthropos; 1985.

19. Pateman C, Agra M. El contrato sexual. Barcelona: Anthropos; 1995.

20. Beauvoir S. O segundo sexo. Río de Janeiro: Sérgio Milliet; 2009.

21. Cobo R. Interculturalidad, feminismo y educación. Barcelona: Cataratas; 2008.

22. Femenías M. El género del multiculturalismo. Argentina: Universidad Nacional de Quilmes; 2007.

23. Ladd P. Comprendiendo la cultura sorda: en busca de la sordedad. Santiago de Chile: CNCA; 2011.

24. Unicef. Estado mundial de la infancia 2013: niñas y niños con discapacidad. URL: https://www.unicef.es/publicacion/ estado-mundial-de-la-infancia-2013-ninas-y-ninos-condiscapacidad. [12.02.2018]. 